\title{
Campus Wireless Network Classification for Enterprise Adoption: Perspectives and Dimensions for Large Scale Computing
}

\author{
E.O. Nonum \\ Dept. of Computer Science \\ Maths, \\ Novena University Ogume, \\ Delta State, Nigeria
}

\author{
P.O. Otasowie \\ Dept. of Electrical \\ Electronic Engineering \\ University of Benin, \\ Benin City, Nigeria
}

\author{
K.C. Okafor \\ Federal University of \\ Technology \\ Owerri, Imo State, Nigeria
}

\begin{abstract}
Owing to large scale computing using the cloud, services can be provisioned with reliable QoS performance. Existing Campus Wireless Network Nigerian tertiary instutions have various challenges in terms of performance, ease of integration, and cost effectiveness. In this paper, a distinct classification of functional network models, their attributes and characteristics was highlighted. Research gaps were identified and a proposed autonomic web services architecture that manages both the performance of service users and the interconnection of WiMax-Wifi infrastructure into a service overlay network was discussed. This represents an intelligent Campus Wireless Network Architecture for services convergence. Low scale and medium scale computing networks were also discussed while outlining their issues. For the large scale computing network model, the advantages of the system include: easing of management tasks through the autonomic systems, ability to self-configure, self-optimizeze and self-heal. Efficient resource management, virtualization and WiMax connectivity interfaces are the core features. This research is still on-going but have developed a workable system for both service deployment and migration without overhauling the generic platforms in existing systems. In the long run, Nigerian instutions will benefit from the improved newtork resilience with improvement in overall performance.
\end{abstract}

\section{Keywords}

Campus, Wide area Network, Wifi, Access Point, Throughput, Latency, Enterprise servers

\section{INTRODUCTION}

Most universities in Nigeria today are yet to fully optimize the gains of a large scale computing network such as the cloud or cluster computing network paradigms. These networks offers data-intensive services such as online application provisioning- multimedia lecturing, e-payments, library, collaboration, etc. The use of existing hotspot networks [1] that uses Wifi Ethernet technologies [2] have various issues ranging from co-channel interference due to microcell access points, coverage and scalability issues, security vulnerabilities, etc. These makes this type of network inefficient for cloud based university services. Again, the generic hotspot networks based on IEEE 802.11 series lacks integrated intelligence for performance provisioning, QoS delivering and in most cases suffers from interoperability problems. Actual performance results greatly vary particularly with (a) line of sight issues; (b) Fresnel zone issues; (c) towers heights (d) noise floors; and: (e) and spectrum issues such as interference. In most cases, these institutions lack adequate knowledge on the type network to adopt for their processes. However, some countries are switching over to improved network paradigms. For instance, in Ethiopia, there is a proposed framework that will utilize the existing IT infrastructure for institutions called Ethiopian Universities Hybrid Cloud (EUHC) [3]. In such a situation, this type of computing network could leverage the capabilities of large scale computing model which combines the local network infrastructure and public clouds.

In a typical Campus Wireless Network, some of the main features required for deploying cloud-based educational content services include: 1) a cloud platform that provides an infrastructure for the realization of a cloud-based educational media service environment, 2) a compatible file format that enables it to provide media content through various types of devices, 3) an authoring tool that enables teachers to create various types of media content, 4) a content viewer that displays different types of media on multiple platforms, 5) an inference engine that provides students with individualized learning content, 6) a security system that manages privileged user access and data encryption in the cloud for dependable educational content services, 7) a WiMax connectivity from users to the backend servers, and a robust datacenter infrastructure.

CWN based on large scale computing could be leveraged to boost education owing to its potential for delivering economical, securable, reliable, and sharable education services [5]. An increasing number of universities and educational institutions in the USA and UK are adopting large scale computing as it can improve on cost savings as well as improve the efficiency and convenience of educational services [6]. A number of studies have been conducted investigating the benefits of large scale computing for elearning systems [7],[8] and have suggested solutions for cloud computing based e-learning systems [5],[ 8], [9].

However, existing hotspot network in Nigerian tertiary institutions focus only on providing poor internet services. Delivering, sharing of learning materials, supporting and establishing an integrated, total cloud-based educational service environment is practically lacking.

Consequently, these forms the basis an on-going research termed On-demand CWN. In the proposed architecture, QoS profiling will be incorporated into a structured distributed user peer-to-peer cloud network that processes autonomic web services. This architecture is inherently a two tier-hierarchical and distributed model supporting Service Level Agreement (SLA) compliant for deployed end user application services. This research will also simplify the system management by 
adhering to autonomic principles of system decomposition while guaranteeing the performance of the system. SLA compliance and services migration to preserve QoS will be shown. Fig 1 shows a typical education context needing a good network computing model.

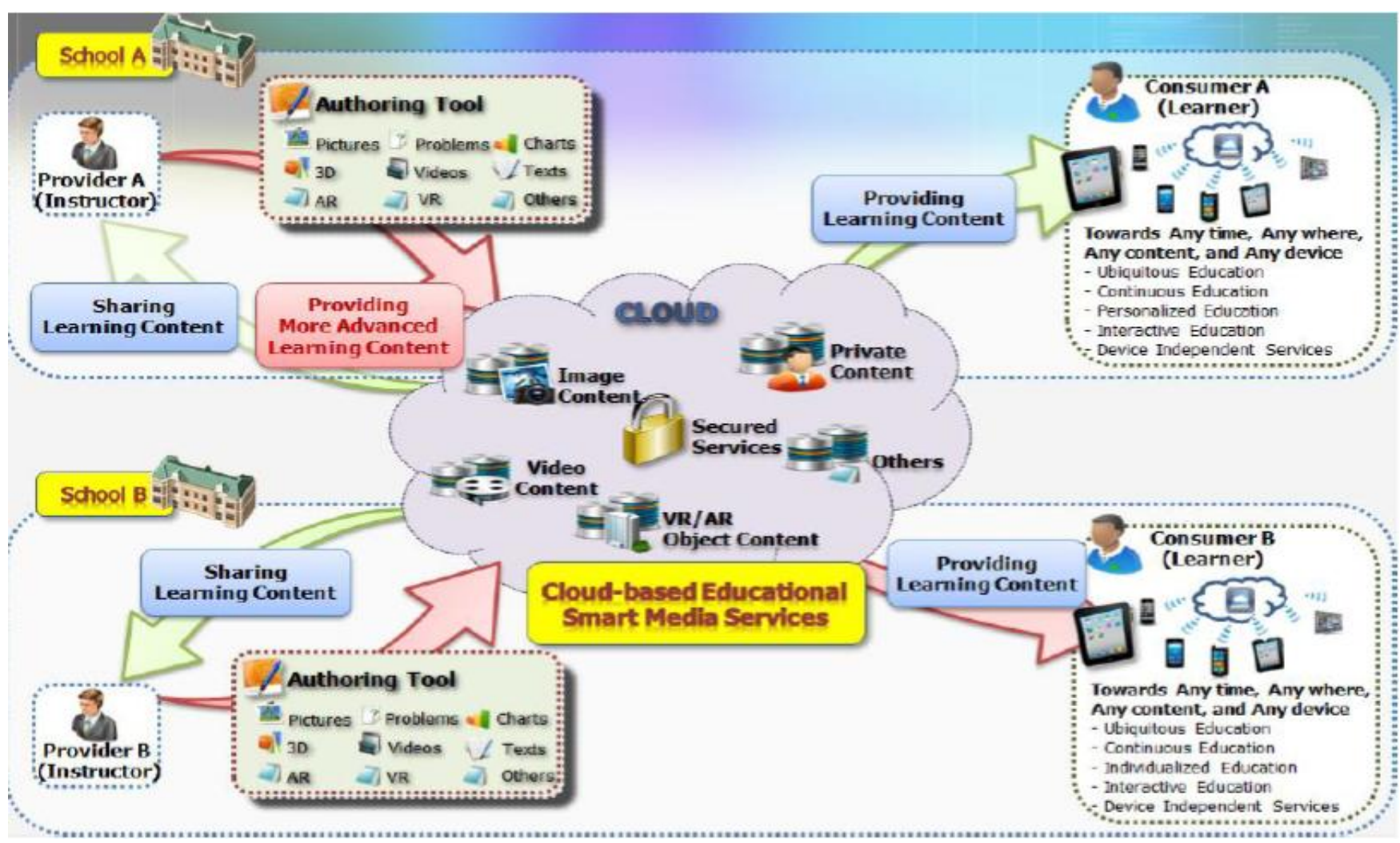

Figure 1. Architecture of a cloud-based education system for smart content services [4]

In this research, the major aim is to derive a complete classification of computing network systems. The work then propose a content-oriented smart educational CWN using WiMax driven cloud computing that integrates a number of features; these features are required to implement a functional cloud-based educational media service environment. The proposed CWN system can enable Nigerian tertiary institutions to deliver and share a variety of enhanced forms of educational content, including texts, pictures, images, videos, 3-dimensional (3D) objects, and virtual scenes of virtual reality (VR) and augmented reality (AR). Esentially, the system consists of six main features required for the deployment of cloud-based educational content services. These include: a two tier cloud based Wifi-WiMax network, WiMax spatial diversity, pathloss model, service provisioning at network core involving resource management and virtualization. These will yield a cost effective CWN.

This paper believes that a well-articulated computing network for CWN services will provide a new and innovative solution for cloud-based educational media services by supporting a cloud-based service environment with a totally integrated system.

The rest of the paper is organized as follows. Section II describes the low scale, medium and large scale network computing models, their characteristics as well as the research gaps. Section III presents the proposed WiMax driven cloudbased education system for smart media content services, and finally in section IV, the work summarizes and conclude the paper while presenting future directions.

\section{LITERATURE REVIEW}

In this section, various works will be reviewed based on three major network classifications viz: Low Scale Network Computing Model (LSNCM), Medium Scale Network Model (MSNM) and Large Scale Network Model (LSNM). Consequently, the research gaps will be enumerated comprehensively.

\section{A. Low Scale Network Computing}

\section{- Autonomous and Light Weight WLAN}

In [10], two models were proposed for choosing the IEEE WLAN architecture. These are the Lightweight WAPs, which form part of a centralized WLAN architecture, having limited functionality, with most of the wireless intelligence residing at a central controlling device (i.e., the WLAN controller). The autonomous architecture uses distributed Wireless Access Points that usually do not require a wireless controller. An autonomous, or distributed, WLAN architecture does not include a wireless controller. In contrast, the lightweight WLAN architectural hardware consists of reducedfunctionality WAPs that operate together with a centralized wireless controller. The controller resides deeper in the LAN, at the distribution or possibly the core layer. Their diffeences boarders on migration scalability, cost and network density. All wireless network are structured based on either of these.

\section{- IEEE 802.11 Standards}

So many wireless networks have been implemented using IEEE 802.11 standard. In [11], the authors carried out a review and summarized the emerging wireless broadband technology i.e. IEEE 802.11. This form of network computing is a set of physical layer standard for implementing wireless 
local area network computer communication in the 2.4,3.6, 5 and $60 \mathrm{GHz}$ frequency band. The standards reviewed include: EEE 802.11a, IEEE 802.11b [2], IEEE 802.11c, IEEE 802.11d, IEEE 802.11e, IEEE 802.11f, IEEE 802.11g, IEEE 802.11h, IEEE 802.11, IEEE 802.11j, IEEE 802.11k, IEEE 802.111, IEEE $802.11 \mathrm{~m}$, IEEE 802.11n, IEEE 802.11o, IEEE 802.11p, IEEE 802.11q, IEEE 802.11r, IEEE 802.11s, IEEE 802.11t, IEEE 802.11u, IEEE 802.11v, IEEE 802.11w, IEEE 802.11x, IEEE 802.11y, IEEE 802.11z, IEEE 802.11ad/ WiGig etc . From all these standards, two operating modes in IEEE 802.11 were identified viz:

i. Infrastructure operating mode- In this case, the STAs communicate with each other through the Access Point. In this scheme, an STA needs to be connected to an Access Point in the network in order to talk to another STA. (Fig.2a).

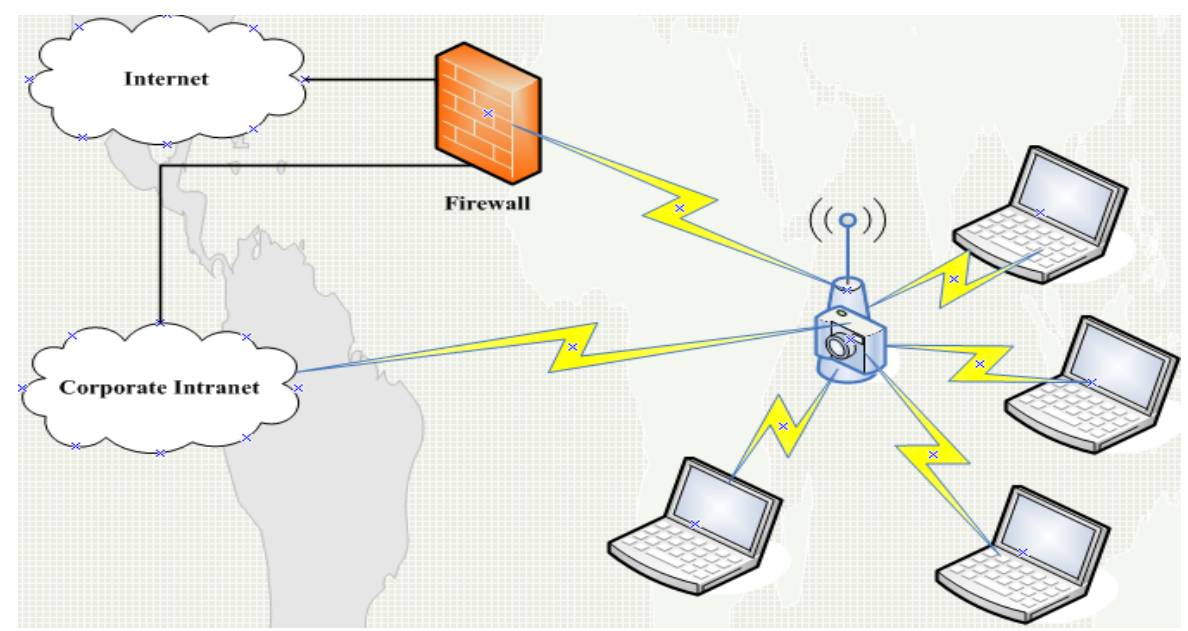

Figure 2a: Infrastructure Mode

ii. Independent operating mode- In the independent mode or ad hoc mode, the STAs can directly communicate with each other. In this mode, the source STA forms an ad hoc link directly with the destination STA (Fig.2b).
Fig 3 shows the newest extended SEE-Mesh and Wi-Mesh proposals.

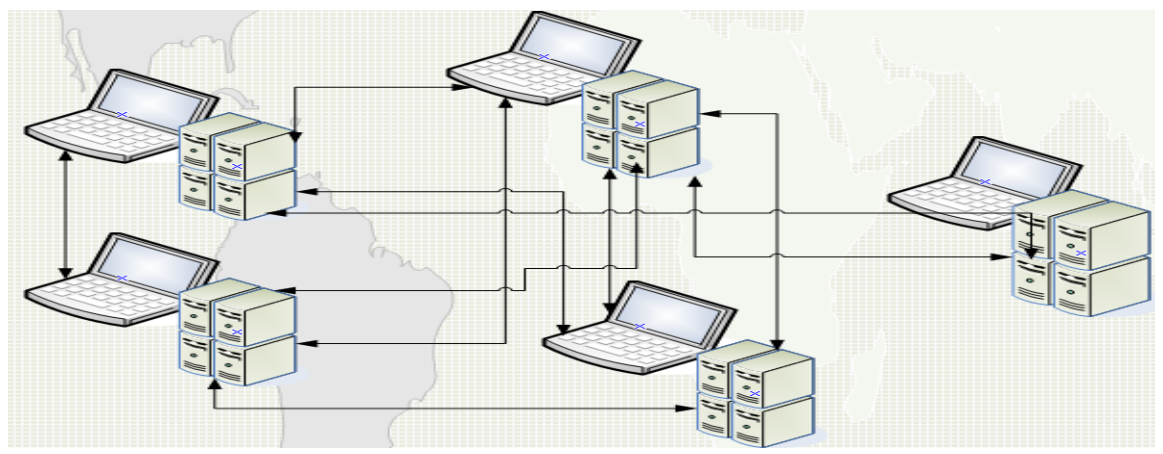

Figure 2b: Independent Mode

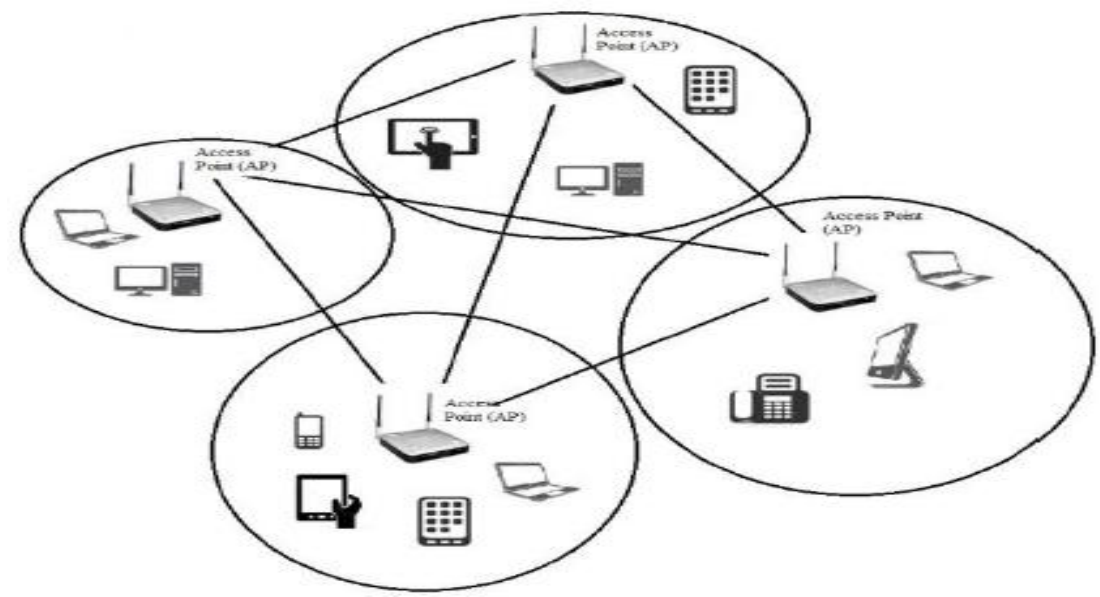

Figure 3: Extended Mesh Configuration Mode [11] 
The issues with these networks include security vulnerability, QoS low data rate and poor MAC layer throughput. The future enterprise Wifi-network computing model will discussed as a way out later in this paper.

\section{- $\quad$ Existing Wifi-Hotspot Networks}

i. Interoperable WiFi and Cellular Networks

The work in [12] proposed an interoperable Wi-Fi hotspot and wide area cellular networks that seeks to maximize user satisfaction and system performance. Their system architecture shown in Fig 4 consists of a set of Base Stations (BSs) belonging to a Packet Cellular Network that can provide wide area coverage to the Mobile Stations (MSs), and a set of Wi-Fi Access Points (APs) that provide high speed connectivity to the MSs. Each MS was assumed to support only one wireless interface that can switch between the packet cellular and Wi-Fi modes of operation. The Wi-Fi APs were

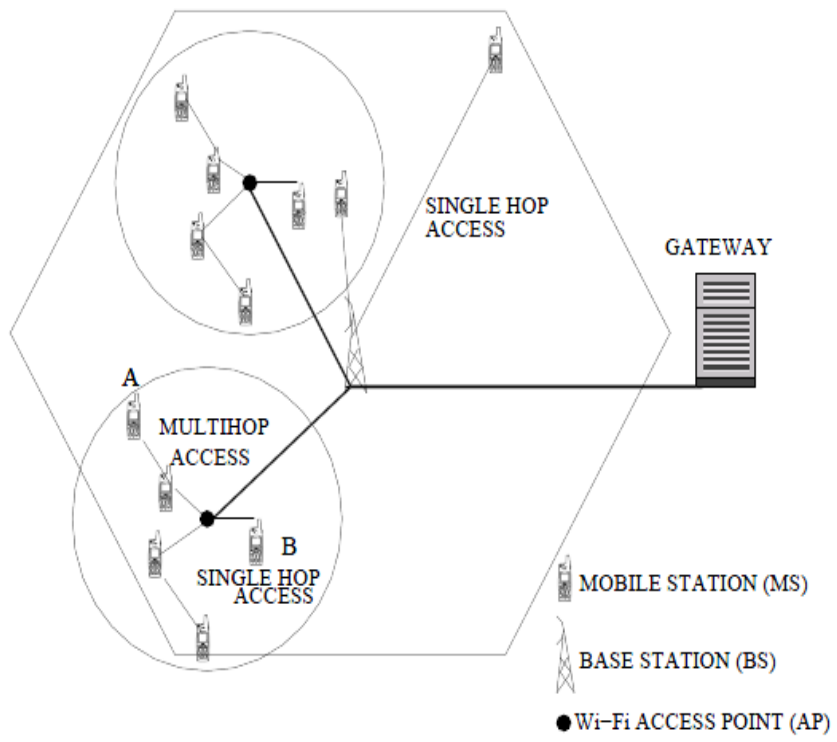

Figure 4a: Interoperability Framework

From Fig 4a it can be deduced that each MS has the option of operating either under the control of the BS or under the control of the Wi-Fi AP. The issues of resource management and services integration were not discussed in relation to QoS for a typical CWN.

\section{ii. GRID WLAN Framework}

In [15], a conceptual high performance hotspot solution termed GRID WLAN was proposed for individuals with PC, notebook, mobile phone or PDA that is Wi-Fi-enabled so as to use its hotspot services (See Fig 5). This have similar architecture with [12]. Their network comprises the infrastructure gateway (ADSL modem and GRID WLAN assumed to be interconnected with one another, and with the BSs by means of either a wired backbone network, or by high bandwidth point-to-point wireless links. The BSs are connected by means of a high speed wired network. The BSs are placed such that the entire terrain is covered, while the $\mathrm{Wi}$ Fi APs are assumed to be randomly distributed throughout the metropolitan area. Their packet cellular network was represented by a Single hop Cellular Network (SCN), in which the MSs are in communication with the BS on the control as well as data channels, with a transmission range equal to that of the cell radius. The Wi-Fi hotspots are considered as multihop relaying environments similar to the Multi-hop Cellular Networks described in [13] and [14]. The $\mathrm{Wi}-\mathrm{Fi} \mathrm{AP}$ acts as the coordinator for enabling routing and reserving bandwidth for MSs in the hotspot. Fig 4a shows the proposed interoperability framework while Fig $4 \mathrm{~b}$ shows the different user profiles

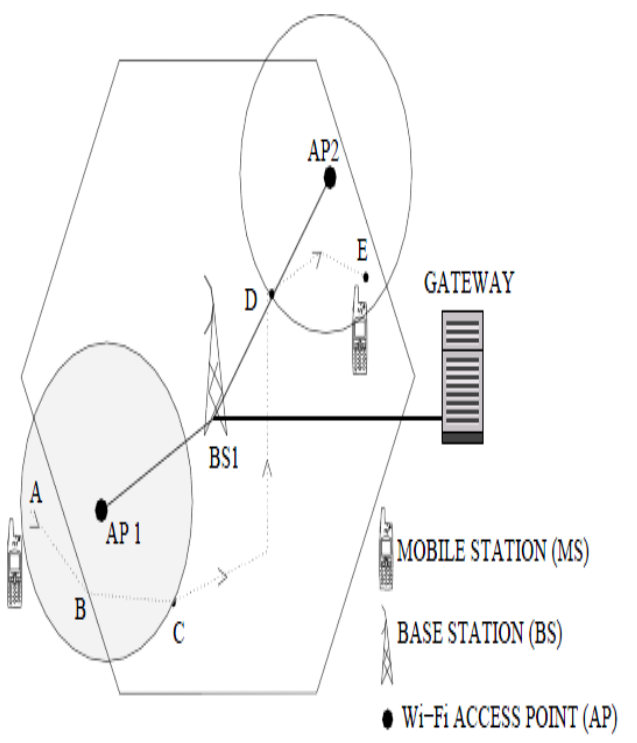

Fig 4b: Different user profiles

Gateway, Hypervisor layer Virtualization firmware), GRID WLAN switch/load balancer, GRID WLAN Access points $\left(\mathrm{AP}_{1} \ldots \ldots . . \mathrm{AP}_{\mathrm{n}}\right)$, Management framework, Wimax base station, VOIP, and hotspot nodes. The network serves as an extended service set GRID WLAN mode. The APs establishes connections to other users who are directly connected to the hotspots. Basically, FTP traffic and HTTP traffic take place in the GRID WLAN hotspot zone sites while the GRID WLAN gateway access point creates a gateway link to the IP cloud (internet). Buffer sizes, loads as well as fragmentation threshold of the GRID WLAN were investigated in their work. 


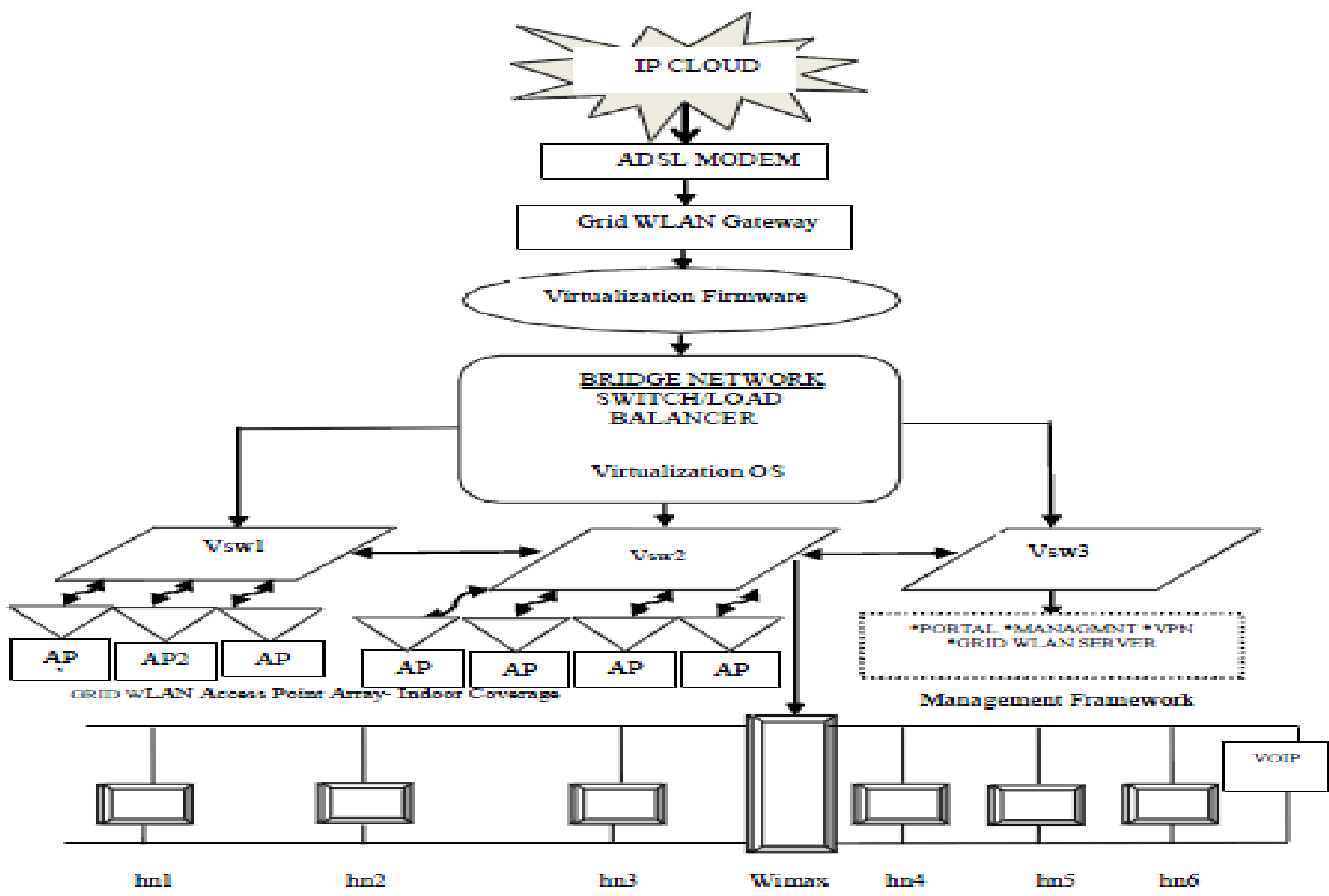

Figure 5: GRID WLAN Architecture [15]

Though the hotspot model fits into the CWN proposal, but similar issues of link connectivity metrics, resource management and services integration were not discussed in relation to QoS for a typical CWN.

iii. Campus General Network

In [16], the authors contextualized the problems of existing campus general network which uses Wireless Access Points (AP). The network model is shown in Fig 6 where poor connectivity to WiFi network at certain areas is one of the major problems. In the network, users have either a Personal Computer or Laptop. Fast connection

to the campus real time system as well as to the Internet are their expectations. With the Access Point deployment alongside various network devices, the networks still have various QoS issues. Most institutions in Nigeria currently uses this type of network architecture.

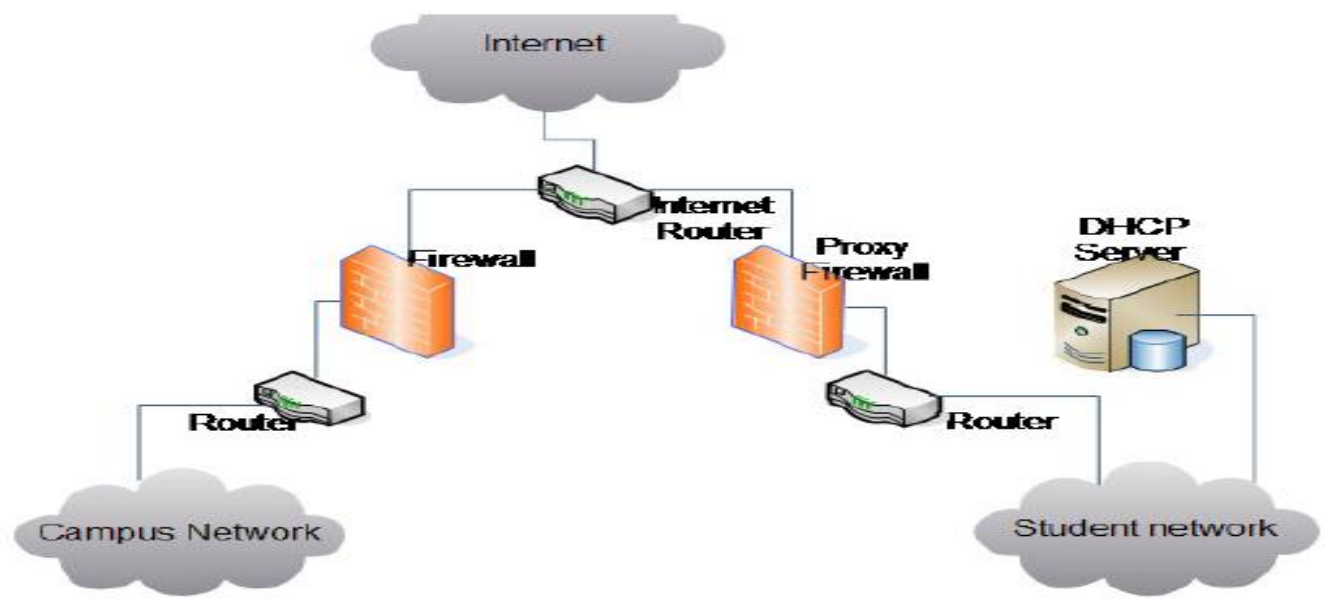

Figure 6: Campus General Network [16]

iv. UNIZIK Hotspot/WLAN IEEE 802.11

In [17], the authors used Nnamdi Azikiwe University-Unizik, Awka as testbed to carry out a study on hotspot/WLAN IEEE 802.11 deployments while devising a cost effective coverage plan in $2.4 / 5 \mathrm{GHz}$ frequency band. A mathematical model on cost optimization for WLAN Hotpot project processes was developed using linear programming, the installation procedure, coverage plan based on specifications of the 
deployment hardware, and data security were covered in this work. Consequently, from the model, the work concluded that with careful selection of optimization criteria in the deployment, an efficient design cost plan, and QoS, could eliminate possible trade-offs in the deployment contexts by over $95 \%$. This network have similar issues with the work in [16].

\section{v. MikroTik Router Hotspot System}

In [18], the authors discussed their usage of MikroTik Router Operating System (OS) to build an authentication and billing System. Their case study was Federal University of Technology, Minna. Like many other hotspot model, this kind of system is used to create security, billing and administration of users on a network connected to the Internet. The network interaction, capacity provisioning, resource management and network coverage issues were not addressed in their work.

\section{vi. RTC-GWLAN/ IEEE 802.11 Hotspot System}

In [19] a discussion on Real Time characterization of IEEE 802.11 hotspot production environment using the University of Nigeria Nsukka (UNN) as a case study was carried out. The research investigated on generic Wireless Local Area Networks (IEEE $802.11 \mathrm{a} / \mathrm{b} / \mathrm{g}$ ) in the context of their Quality of Service $(\mathrm{QoS})$ in high density networks using some generic Key Performance Indicators (KPI) such as throughput (Bytes/Sec), Received Signal Strength Indicator (RSSI), and latency response (secs). The work made deductions from traditional (Generic) Hotspot Testbed while outlining the limitations of the generic hotspot network. However, the work did not propose any network model that will address the existing deficiency in CWNs.

B. Medium Scale Networks

- Non-Cloud Based WiMax Hotspot Networks

Several studies and analysis on Wimax techonology have been studied in [19],[20],[21],[22], and [23] with emphasis on QoS in Wimax based network systems. From the works, it was agreed that WiMAX is the solution of "last mile" wireless broadband and provides an enhanced set of features with flexibility in terms of potential services. Some of the highlighted features include [24]:

interoperability, high capacity, wider coverage (it supports the different modulation technique constellations, such as BPSK, QPSK ,16-QAM and 64-QAM), portability, non-line-of-sight operation, higher security, very high peak data rate, adaptive modulation and coding (AMC), link-layer retransmission, quality of service support and ip-based architecture. WiMAX provides multiple flexible architectures such as Point-toMultipoint, Ubiquitous Coverage and Point-to-Point. In the context of Wimax Hotspot networks, the research efforts are discussed below.

In [25], [26], the authors explored the Wi-Fi and WiMAX technology, its operation and the deployment contexts. The work carried out a comparison of both networks while showing that Wimax performs optimally. The work identified the challenges in wireless networks which include issues like security, seamless handover, location and emergency services, cooperation, and QoS but failed to address these issues. Figure 7 depicts the usage of handheld devices in mobile, portable and public hotspot environments [27]. In [27], the paper explored the complementary nature of WiMAX and WiFi, as well as illustrates how service providers can leverage these technologies to offer wireless broadband Internet connectivity and compelling new services at affordable prices and in more locations. It also focuses on the synergies between the IEEE $802.11 \mathrm{a} / \mathrm{g} / \mathrm{n}$ Orthogonal Frequency Division Multiplexing (OFDM) and IEEE 802.16e-2005 OFDMA air interfaces. The paper lacks description on resource management for grid services.

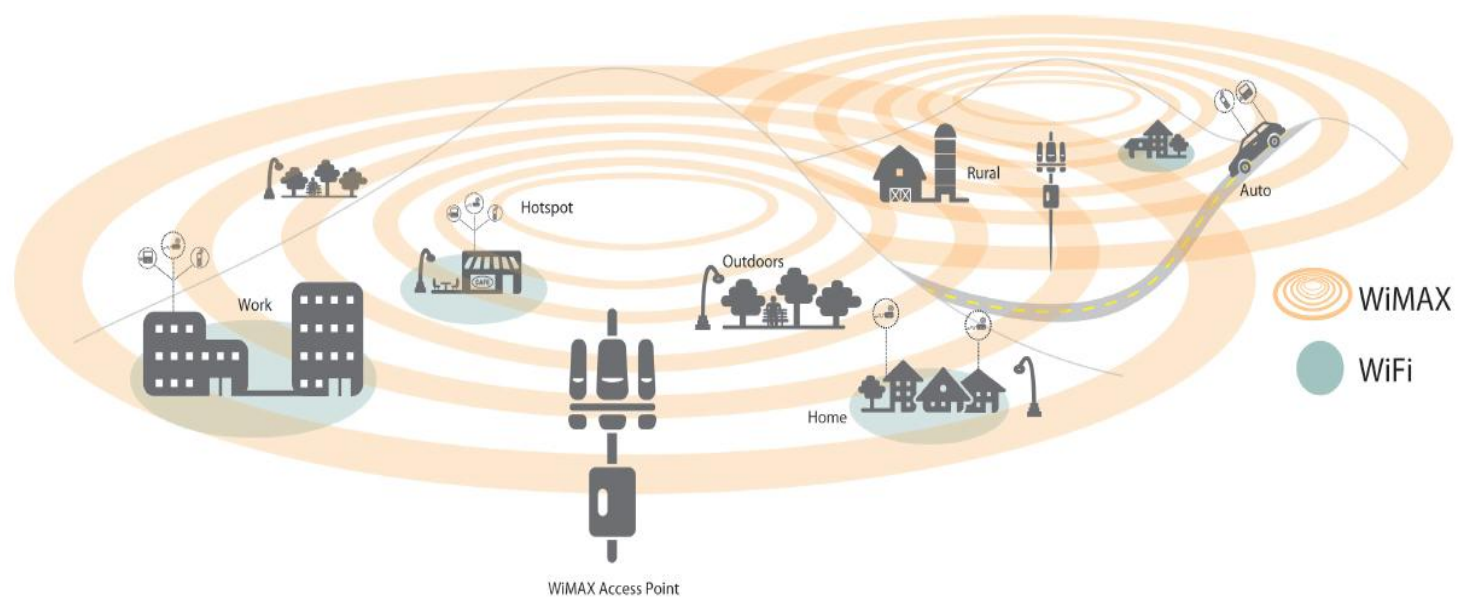

Figure 7: Usage of handheld devices in mobile, portable and public hotspot environments [27]

- Enhanced Enterprise Wifi-Wimax Model for Medium Scale Network Computing

Cloud Based Wifi-Wimax Networks based on two tier data center model is the proposed direction for CWN. With Triband WiFi, Light Fi (LiFi) wireless solutions [28] whose network topology offers data rate increased to $5 \mathrm{Gbps}$. The aim is to utilize these networks in enterprise contexts to get increased wireless speed, improved wireless access and increased network capacity. By leveraging the enterprise wifi standards, major improved Wifi technologies such as WiFi
Gigabit solution (IEEE802.11ad or WiGig standard), White Fi solution (IEEE 802.11af standard), Wi-Fi approach for Machine to Machine (M2M) communication (IEEE 802.11 ah standard), HetNets solution, Cognitive-Fi (CogFi) can be improved for cloud based computing network. By these network topologies, the data rate can be increased to $5 \mathrm{Gbps}$.

Consider a Long Term Evolution (LTE)-WiFiHetNet solution which have a single base station (BS) and many number of $\mathrm{WiFi}$ access points (APs). This can provide service to a large 
but homogenous coverage area. Fig 8 shows such typical network architecture which provides a mixer of networks (LTE and Wi-Fi network) implemented in hierarchical levels. The coupling elements are connected in between routers and Wi-Fi access points. This junction of the network is highly integrated while still allowing another coupling element that connects internet access with WiFi access point. The backend servers must be optimized for high performance computing in order to deliver the required QoS.

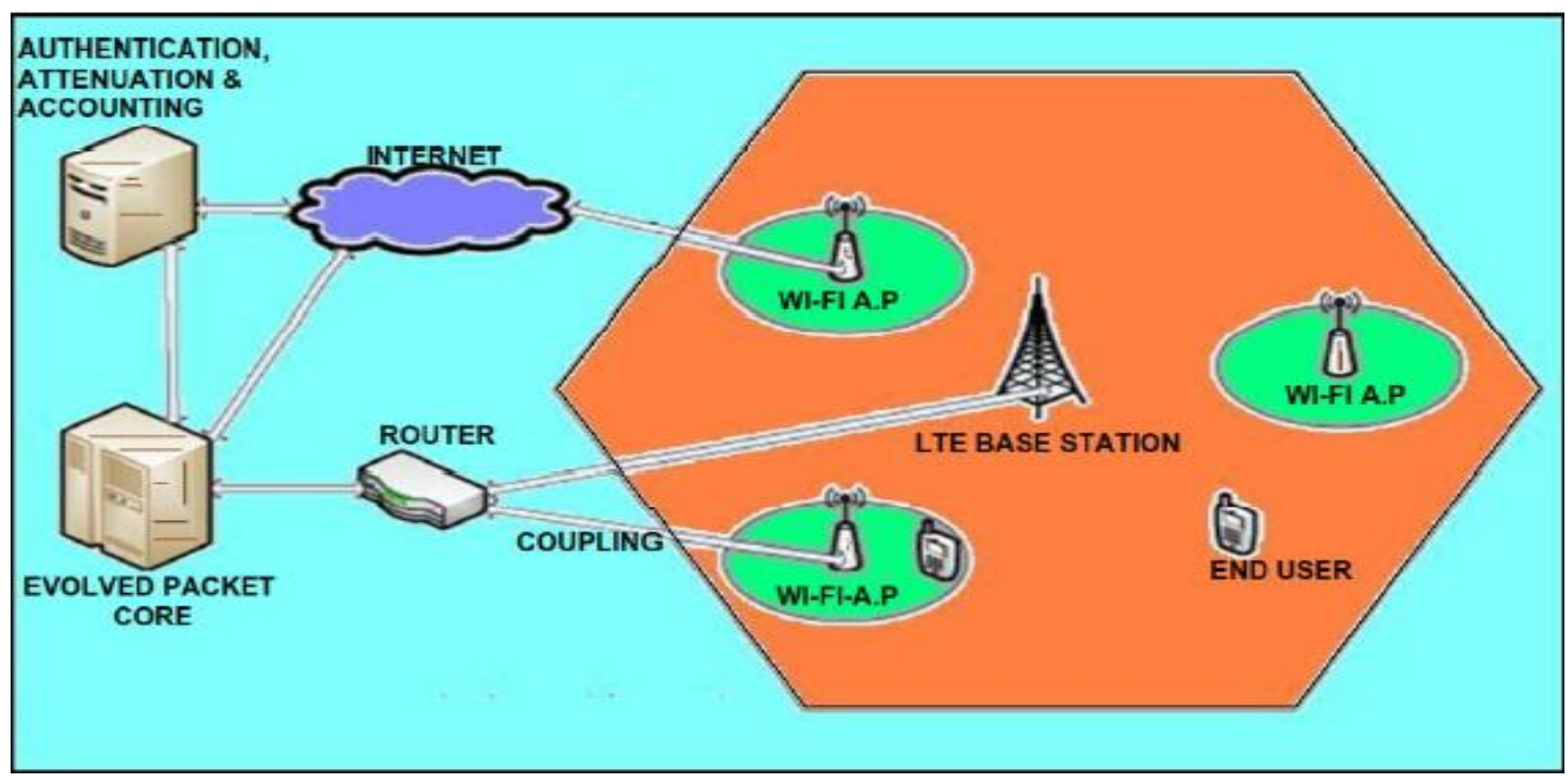

Fig 8:-WiFi Heterogeneous (HetNet) LTE-Network [28]

To achieve multiple interfaces while linking the internet, WiFi, Bluetooth and Zigbee are connected through cognitive network as shown in Fig 9

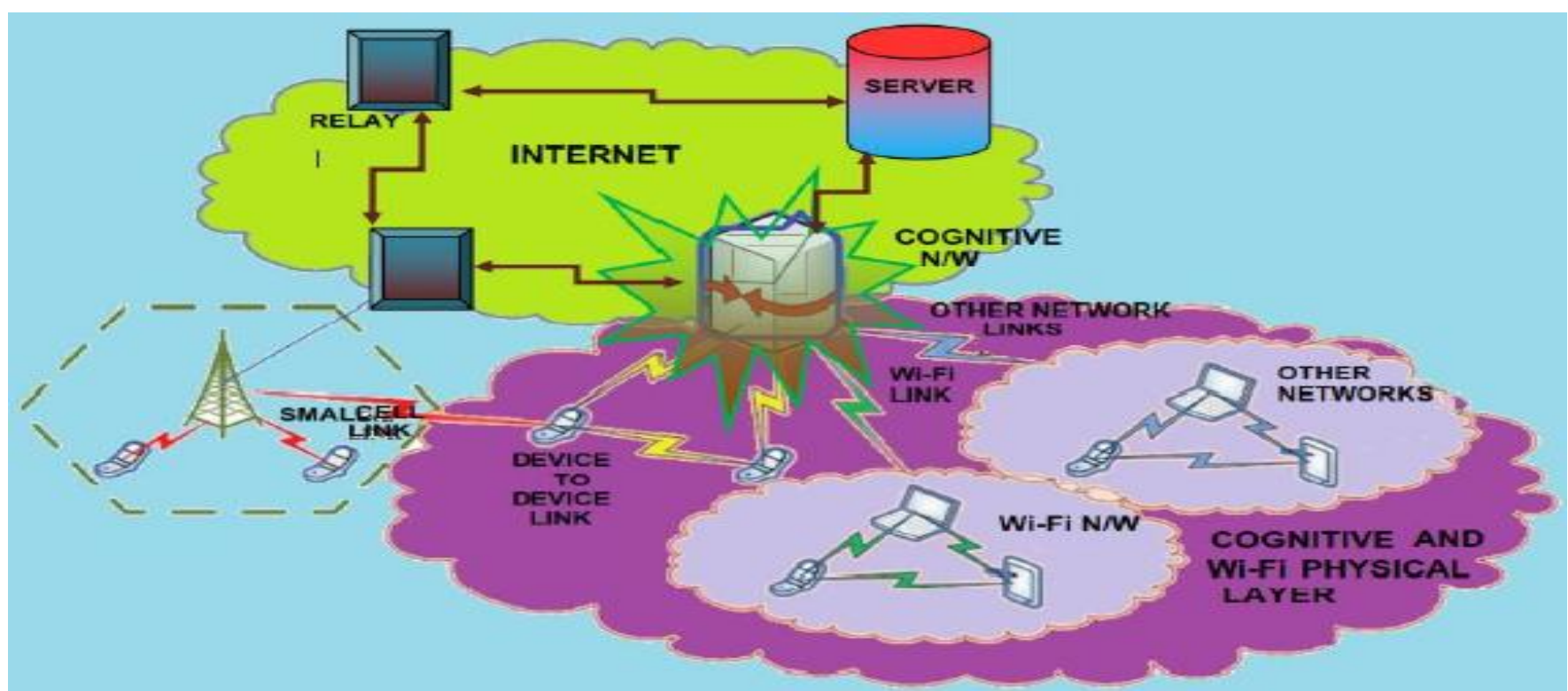

Figure 9: Connectivity Interfaces of Cognitive network with Wi-Fi [28]

As explained previously, the main features of the desired CWN include:

i. Two tier cloud based wifi-wimax networks supporting WiMax spatial diversity with excellent pathloss index

ii. Service provisioning at network core iii. Efficient resource management via virtualization for cost effective design

The anticipated gains of these network standards when fused with cloud computing capabilities include:

i. It will facilitate the expansion of the cloud for small and medium sized enterprises. 
ii. WiFi- based location analysis will help different organization to improve the customers and user WiFi experience, and this will lead to increase in business intelligence

iii. They improve Hotspot 2.0 and pass point open services via its integrated and advanced features

iv. They could allow user to log in to the WiFi network by using their assigned credentials.

v. They support only the $5 \mathrm{GHz}$ frequency, meaning that the signal has less range.

vi. They supports multiple antennas that is MIMO (multiple input multiple output). For instance, the Super Wi-Fi (400 meters) signals can travel over long distances than WiFi (100 meters). It can travel 4 times the $\mathrm{Wi}-\mathrm{Fi}$ as a result more bandwidth, lower network costs and lower power consumption can penetrate two or three concrete walls but Wi-Fi cannot.

vii. Offers greater efficiency due to longer distance coverage, wider range, more bandwidth andlower power consumption.

viii. Super Wi-Fi devices have the ability to switch from one group of channels to another.

ix. Offers advanced and powerful features with selfexplanatory and user friendly interface.

The above reviewed works are still non-cloud based WiMax networks. This work will now discuss the large scale computing network concept for the CWN.

\section{Large Scale Computing Networks}

\section{- Grid/Cluster and Cloud}

Large Scale computing network model is the new paradigm in CWNs. These are networks that leverage the computing capabilities such as grid, cluster utility philosophies yielding the cloud computing service benefits for end users. A number of computing researchers and practitioners have attempted to define clusters, Grids, and Clouds [29] in various ways. This work presents some definitions deemed fit for these classes of networks (clusters/grid) among others, as follows:c

According to Pfister's [30], a cluster is a type of parallel and distributed system, which consists of a collection of interconnected stand-alone computers working together as a single integrated computing resource."

In Buyya's [31], the work defined clusters following the popular definitions for Grids at the 2002 Grid Planet conference, San Jose, USA as follows [32]:

- "A Grid is a type of parallel and distributed system that enables the sharing, selection, and aggregation of geographically distributed autonomous resources dynamically at runtime depending on their availability, capability, performance, cost, and users' quality-ofservice requirements."

- Based on the observation of the essence of what cloud promises to be, the work in [32] proposed the following definition:

- "A Cloud is a type of parallel and distributed system consisting of a collection of inter-connected and virtualized computers that are dynamically provisioned and presented as one or more unified computing resource(s) based on service-level agreements established through negotiation between the service provider and consumers." At a cursory glance, Clouds appear to be a combination of clusters and Grids". However, this is not the case. Clouds are clearly next-generation datacenters with nodes virtualized through hypervisor technologies such as VMs, dynamically provisioned on demand as a personalized resource collection to meet a specific service-level agreement, which is established through a negotiation' and accessible as a composable service via Web Service technologies such as SOAP and REST [32].

Example of such networks have been described in DCCN/EETACP [33] (IaaS - PaaS), CycloidGrid [34], Manjrasoft Aneka (IaaS - PaaS) [35], Amazon EC2 (IaaS), Microsoft Azure (IaaS - PaaS), TeraGRID [36], ESciencE, (EGEE) [37], Google App Engine (IaaS - PaaS) [32], etc. Several works on cloud networks have been studied in [29], [31], and [33], however, by enhancing the capabilities of the existing Wifi/Wimax networks, a high performance CWN will be evolved. This is the aim of this research. This network is referred to as cloud Based Wifi-WiMax network model. This satisfies the requirement of large scale computing for campus services and grid collaboration.

\section{- $\quad$ Limitations of Traditional Enterprise Networks}

From the findings in literature, this work summarised the limitations of traditional CWN as follows:

\section{i. Scalability}

The rapid growth of online/web services and applications that runs on a cloud data center networks makes scalability a key design factor for the existing DCN and CWN.

Most traditional CWN are not scalable, hence, when the number of web application or online users of such network increases, the network will either be congested or break down entirely. A scalable cloud based CWN has the capacity for incremental expansion by adding more servers into the already operational structure without affecting the performance of the already existing running servers.

\section{ii. Network convergence and downtime}

Traditional hotspot networks have problems of network convergence and downtime thereby disrupting operations as evidence by most users on these network. If a system becomes unavailable due to downtime, critical network services may be impaired or stopped completely. It is necessary to provide a reliable infrastructure for CWN operations, in order to minimize any chance of disruption.

iii. Latency

This is the amount of time that it takes for a packet to be transmitted from one point in a network to another point in the network. In relation to the traditional $\mathrm{CWN}$, the overall architecture generates over 30\% network latency in switching and traffic delay thereby negatively affecting responsiveness to business demands and services. This is because some of them were not based on technologies such as virtualization which drastically reduces the number of network devices.

\section{iv. Throughput Performance}

Throughput is a term used to describe the capacity of a system to transfer data. Since the demand for data exchange in Enterprise networks is extremely large compared with other networks, the first design goals is to maximize the throughput. 
The amount of bandwidth allocated to different types of packets affect throughput. Due to the bulky architecture of the traditional data center networks which results to about 30 percent network latency in switching, the overall throughput is negatively affected.

\section{v. High infrastructure Economy}

The cost of deployment as well as maintaining their infrastructure in generic CWN is very immense and hence calls for a better approach to cost reduction as well as service availability.

\section{vi. Network Congestion Effects}

The existing networks creates unnecessary bottlenecks and congestion particularly with poor bandwidth subscription for the users on the link.

In this context, the goal of this research is to design a campus network communication architecture that meets the following goals:

i. Addressing radio propagation issues such as signal fading, and Bit errors, etc

ii. Scalable interconnection bandwidth: it should be possible for an arbitrary host in the datacenter to communicate with any other host in the network at the full bandwidth of its local network interface.

iii. Economies of scale: just as commodity personal computers became the basis for large-scale computing environments, we hope to leverage the same economies of scale to make cheap off-theshelf Ethernet switches the basis for large scale data center networks.

iv. Backward compatibility: the entire system should be backward compatible with hosts running Ethernet (IEEE services) and IP. That is, existing datacenters, which almost universally leverage commodity. Ethernet and run IP, should be able to take advantage of the new interconnect architecture with no modifications.

v. Highly intelligent with dynamic fault tolerance will facilitate campus services network capacity optimization design for tertiary institutions

vi. Highly cost effective when considering the scale of computing in today's network infrastructures for similar purposes.

Developing a Cognitive Campus Wireless Network with Wimax Spatial diversity capable of supporting high density network services such as cloud computing services, mobile video, mobile audio etc remains the focus of this research

D. Research Gaps

i. Today's network design, control and traffic management reflecting in QoS while addressing the aforementioned limitations in the generic CWN have not been addressed.

ii. These generic networks are highly undesirable, since availability and traffic delivery requirements are not guaranteed.

iii. Development of dedicated wireless architecture for intelligent CWN offering low-latency facilities network using low-cost, $60 \mathrm{GHz}$ beamforming radios have not been fully explored. iv. A CWN that provides a robust paths decoupled from the wired network, while offering the flexibility to adapt to workloads and network dynamics have not been addressed.

v. Resource management utilizing virtualization in generic CWN have not been explored.

Having explored the various network classifications, attributes, merits and limitations, the development of a novel CWN that takes into cognizance hotspot traffic engineering vis-à-vis intrinsic network behaviour and extrinsic radio propagation is proposed and the intelligent $\mathrm{CWN}$ is detailed next.

\section{PROPOSED SYSTEM}

- Characterization of Intelligent Campus Wide Network Architecture

Following the issues from the existing CWN, this research seek to develop a more robust, scalable and flexible CWN that will support services integration and application convergence while offering the best quality of service to end users. The network seeks to offer solutions that will address challenges in link coordination, link interference and network failures. The testbed measurements and simulation results show that the proposed model enables large number of low-latency control paths to run concurrently, while providing low latency end-toend message delivery with high tolerance for radio and rack failures.

In this work, a consideration of the novel possibility of using high performance WiMax AP links into a cloud data center (DC), to augment the capabilities of the generic CWN was made. This is a promising approach to explore for several reasons. First, it is note that the machines in the generic CWN server farms are densely packed, so wireless devices that provide high bandwidth over short ranges are a natural fit. Second, the radio environment is largely static since people and equipment move around infrequently, minimizing fluctuations in wireless link quality. Third, line-of-sight communication is achievable by mounting $60 \mathrm{GHz}$ radios on top of racks. Fourth, the wireless hotspot network is available as a reliable channel for coordinating wireless devices, thereby simplifying many traditional wireless problems such as aligning directional senders and receivers, and interference avoidance.

Traditional, wired datacenter networks are tree-structured and oversubscribed to keep costs down. For example, a typical DC rack comprises 40 machines connected to a top-of-the-rack (ToR) switch with $1 \mathrm{Gbps}$ links. The ToR is connected to an aggregation switch (to network with other racks) with 10 Gbps links. Thus, the link from the ToR to the aggregation switch can be oversubscribed with a ratio of 1:4. However, each oversubscribed link is a potential hotspot that hinders some DC application. This problem is tackled by combining many more links with OpenFlow switches with variants of multipath routing so that the core of the network is no longer oversubscribed This benefit comes with low material cost and little implementation complexity.

\section{- $\quad$ System Overview of the Proposed Reengineered CWN}

The block diagram model of the reengineered data center network shown in Fig 11 shows the following block components which are: user domain, access/virtualization layer, hybrid speed redundancy layer. They are described as follows. 
i. User Domain Block.

In the proposed system, the user domain is the termination point of the cloud CWN where users with their client machines (workstations, PDAs, PCs, Laptops) can gain access into the network. Owing to virtual machine logic instantiation in the enterprise server, upon authorisation users can make connection and access resources $\mathrm{n}$ the network. Beside the security configurations done at the other layers such as hybrid speed redundancy layer and access layer, high level security is implemented at the user domain since it supports extensive platform security.

ii. Access Layer Block.

Access/Virtualization layer runs on an enterprise server architecture having two layers of caching services, one for virtual machines (VM) outer loop and the other for the servers attached to the VMs and its related applications. This layer is designed to have resilience, scalability, robustness owing to its hardware configurations. Also, beside the caching services, bandwidth optimisation is realised in this layer. Media Access Control (MAC) controllers and servers (database, application and web servers) are the devices located in this layer. This layer allows for terminal connectivity with data center network (CWN) switch. The user domain devices (PCs,
PDAs, Laptops, iPAD and mobile phones) only need to have a compatible remote desktop protocol (RDP), media access control (MAC) which are implemented by MAC controller to be able to connect to a terminal CWN switch.

The server media Access Controller (MAC) implements a combined protocol referred to as Carrier Sense Multiple Access with Collision Detection and Traffic Arbitration Messaging Protocol (CSMA/CD + TAMP)' which makes for an efficient and flexible data throughput while optimizing bandwidth in the proposed reengineered CWN architecture. By connecting to a terminal switch virtual local area network (VLAN), sessions are created which runs and controls user access. Also, programs like anti-spyware, anti-viruses run both on the client machines and high-powered computing virtual machine engine server (VM-server) to protect user data. User data is always stored in a centralized location server other than the user work stations thereby simplifying data and recovery processes. The anti-spyware and the antiviruses are updated from the virtual server ensuring that all the definitions are up to date. Applications are also updated centrally from the virtual server. Robust firewalls are also introduced in the network.

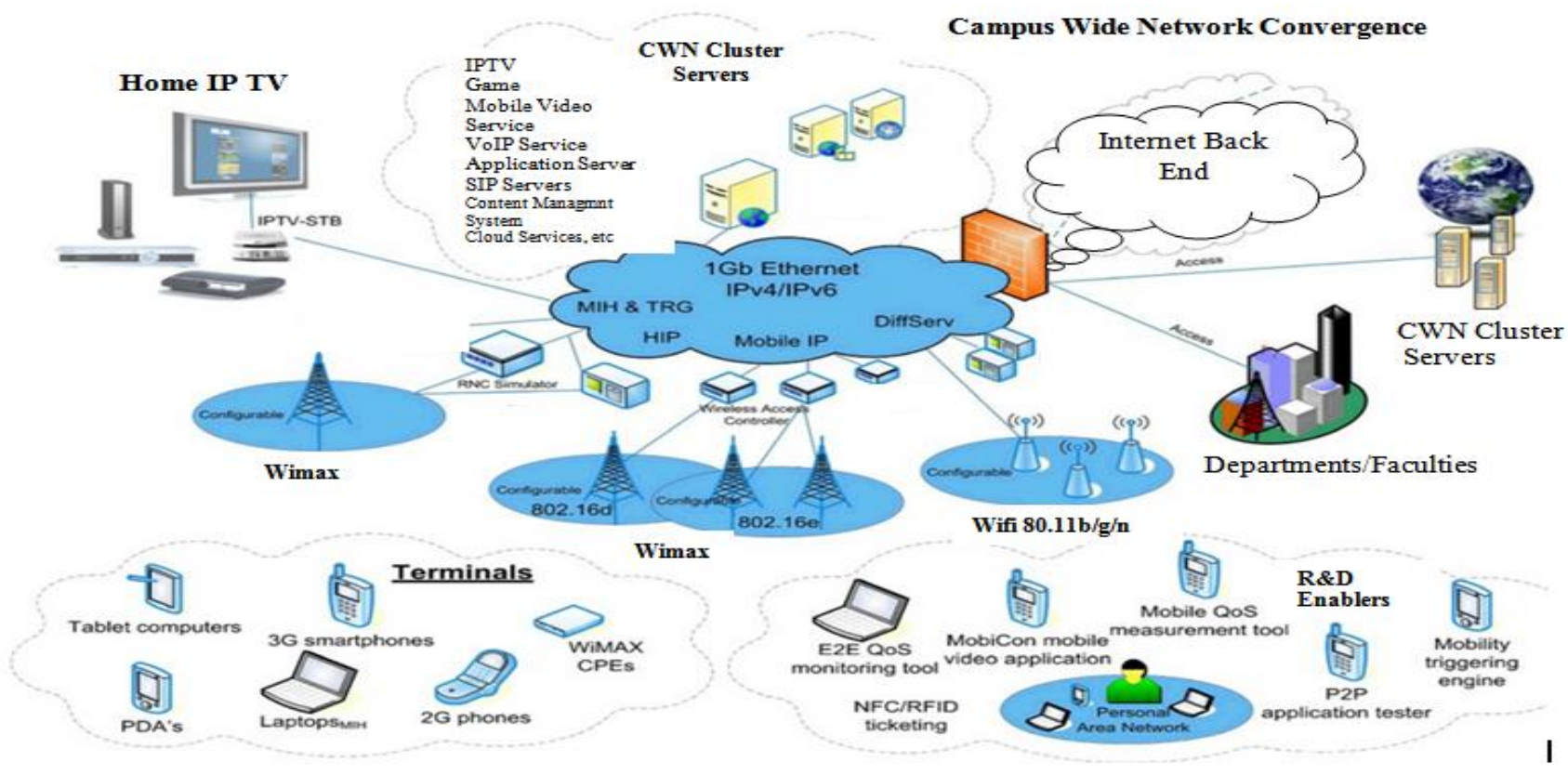

Figure 10: Proposed Intelligent CWN Architecture for Services Convergence

iii. Server Virtualization Module

In this work, VM-server is a high computing Ethernet server deployed to provide remote accessibility and service provisioning to all the client machines in the network. Through the concept of virtualization, various server instances are created, running different services. This virtualized server provides cheap client hardware and having the users connected to it to run more powerful applications such as word processors, internet browsers, e-mail clients. In this work, various server instances were created in the VM box. The server keeps specific user account of the client machines and gives them the ability to access any virtual application or server for the organisation. Audit logs, traces and transaction times are kept by the server. The server has security layer setting used to configure it for authentication which is how it verifies client identity. This allows a secured socket layer (SSL) certificate which enables the client machines to connect. Using this method reduces the risk associated with authentication credential from suspicious users and networks. The encryption on the server has different levels used to protect the content of the server from interception from suspicious parties. On the server, group policies are configured.

Some of these policies include connection policy, device and resource redirection policy, licensing policy, profiles policy, remote session environment policy, security policy, session time limit policy. Finally on the virtualized server, the cloud services are modelled as a transmission control protocol (TCP) http service from the application supported services of the server. Other TCP parameters and IP host processing 
information will be defined based on the critical threshold of operation. The link interface that connects the access/virtualization layer and the hybrid speed redundancy layer is fibre channel over Ethernet (FCoE). FCoE intends to maintain the Fibre Channel protocol over $40 \mathrm{~Gb}$ Ethernet networks by mapping Fibre Channel frames over full duplex IEEE 802.3 networks. FCoE de-layers and simplifies the data center network architecture when it is ratified.

iv. Hybrid Speed Redundancy Layer Block

Fig 11 shows the hybrid speed redundancy layer block. This will be modelled to completely replace the core and distribution layer of the traditional data center network. In other words, we adopted the two-layer data center model owing to its advantages over the three-layer model. High speed switching which will be achieved by the use of multiprotocol label switch (MLS) with VLAN segmentation. This were considered for efficient packet delivery from sources to destinations and vice versa in this layer. Aside it's dual routing and switching functions at a very high speed; MLS has in-built VLAN capability for VLAN segmentation of data center networks. Fig 11 also shows the block diagram of the proposed reengineered CWN capturing the WiMax access point, the air interface and the internet core compute resources.

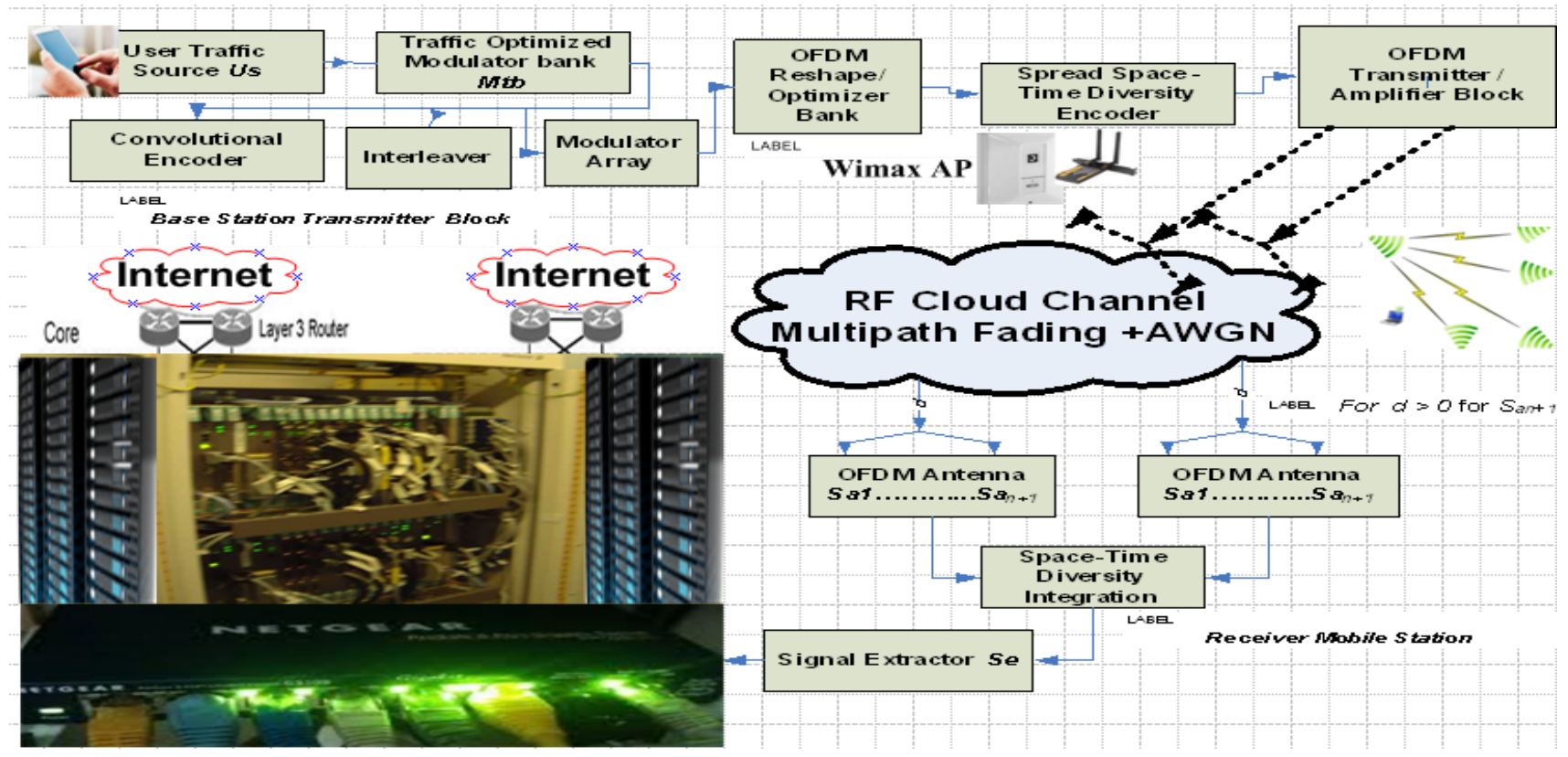

Figure 11: Block Diagram of Generic Communication System with WiMax AP air interface and CWN core

As shown in Fig 11, the CWN model basically leverages the Orthogonal Space Time Block Code Space Diversity (OSTBC) in its architecture. This basic feature will be developed to exploit the multipath characteristics of the channel. The system model is designed for efficient traffic propagation which will address network scalability, efficient modulation, RF spectrum optimization and gain amplification, resulting in lossless traffic propagation. The model will address transmitter base station characteristics, RF cloud channel and multi-tenanted receiver optimization modules reengineered for gain amplification for wireless deployment scenarios. The system, also makes use of the space diversity architecture which seems to have a better features for traffic propagation integration. With its well-defined structure, a high-level WiMax transmitter base station model is constructed from many low-level subsystems at the same level and is fully connected with one another as show in Fig 10 .

For efficiency in space-time diversity integration which is part of our drive for this work; such efficient interconnection of the base station modules provides higher traffic receptivity and good capacity for efficient packet delivery and low bit error rate network. While considering the WiMax base station interconnection concepts in Fig 11, this work will introduce the following into the system model:

- Analytical model that handles traffic propagation issues in the base station.
- Orthogonal Frequency Division Multiplexing model for the Space-time diversity scheme used in the design.

- An RF channel Cloud segmentation for logical isolation of the base station and the receiver in the architecture of the generic wireless communication system.

- A traffic gain OSTBC which is meant to suppress or normalise RF multipath channel effect while being fault-tolerant at the same time.

- Distortion cancellation in the Wimax CWN for broadcast traffic regulation optimization.

In designing the proposed CWN model, the main goals are to maintain good throughput, low BER, low latency errors, and high sensitivity with less administrative overhead. The Wimax CWN should be capable of supporting High Performance Traffic Workloads (HPTW) like voice, video, web applications, cloud services, and data service. Granting that the performance of today's mobile communication systems is measured by QoS parameters such as throughput, latency, and service availability, from the network perspective, throughput, low latency errors, service availability and sensitivity will largely depend on the space-time diversity framework proposed in this research.

The system architecture in Fig 10 is inherently a two tierhierarchical and distributed model supporting Service Level 
Agreement (SLA) compliant for deployed end user application services.

By using resource allocation, scheduling, via virtualization, a simplified management of the system is feasible in the CWN. Also, by adhering to autonomic principles of system decomposition, all the network variables could be identified for inclusion in the overall design. Performance of the system will be based on QoS and SLA compliance.

\section{CONCLUSION AND FUTURE DIRECTION}

This paper has dealt with a proposed large scale computing network leveraging Wimax Cognitive AP physical model, two-tier design, resource allocation and link performance model as part of its design subsystems. This converge into a cloud based network for running services in Nigeria tertiary institutions. Various network computing models were reviewed while identifying the research gaps. This distinct features of the proposed CWN were discussed. The proposed CWN is perceived to be cost effective, flexible and yields a high performance network owing the resource management via virtualization. The network is a cloud integrated networks for autonomic service containers. It is a scalable and decentralised CWN for the deployment of distributed web services was articulated. In this context, the work simplified the management of the system by adhering to autonomic principles. It is believed that user satisfaction on the deployed cloud services in the proposed system will be guaranteed. The work is still ongoing but have the following as part of the future work:

i. To characterize some selected institutions as hotspot testbed case study in terms of latency and throughput using MikroTik, Ethereal Wireshack software for User datagram protocol (UDP) and Transmission Control Protocol (TCP) services on such networks.

ii. To show how spatial diversity, and virtual cell schemes can be used to enhance $\mathrm{CWN}$ performance.

iii. To develop the analytical models for spatial diversity, Wimax recursive virtual cell mapping and other performance QoS models.

iv. To formulate and develop a simple Wimax Cognitive AP physical model while accounting for SNR, Bit Rate and PER under the influence of channelization and fading.

v. Pathloss influence of the Wimax network over Wifi will be carried out also

vi. To carry out performance evaluation of the proposed Wimax cognitive hotspot network using discrete event simulation approach while employing the relevant metrics such as throughput, latency transistion, service availability, delay transition, localization, etc, while comparing with that of the exiting testbeds.

With the developed workable system for both service deployment and migration, without overhauling the generic platforms in existing systems. In the long run, we shall benefit from the improved resilience with improvement in overall performance.

\section{REFERENCES}

[1] Udeze.C.C, Okafor K.C,H.C Inyiama, C. C. Okezie,“A Conceptual Design Model for High Performance Hotspot Network Infrastructure (GRID WLAN), (IJACSA)
International Journal of Advanced Computer Science and Applications, Vol. 3, No. 1, 2012, Pp. 93-99

[2] S. Vasudevan, K. Papagiannaki, C. Diot, J. Kurose and D. Towsley, Facilitating Access Point Selection in IEEE 802.11 Wireless Netwoxrks, USENIX Association Internet Measurement Conference 2005.

[3] Shaik Saidhbi, "A Cloud Computing Framework for Ethiopian Higher Education Institutions, "IOSR Journal of Computer Engineering (IOSRJCE)", Volume 6, Issue 6 (Nov. - Dec. 2012), PP 01-09.

[4] Ji-Seong Jeong, Mihye Kim and Kwan-Hee Yoo, “ A Content Oriented Smart Education System based on Cloud Computing", International Journal of Multimedia and Ubiquitous Engineering Vol.8, No.6 (2013), pp.313$328 \mathrm{http}: / / \mathrm{dx}$.doi.org/10.14257/ijmue.2013.8.6.31.

[5] M. A. H. Masud and X. Huang, "An E-learning System Architecture based on Cloud Computing", World Academy of Science, Engineering and Technology, vol. 62, (2012), pp. 74-78.

[6] N. Sultan, "Cloud computing for education: "A new dawn", International Journal of Information Management, vol. 30, (2010), pp. 109-116.

[7] P. Pocatilu, F. Alecu and M. Vetrici, "Using Cloud Computing for E-learning Systems", Proceedings of the 8th WSEAS international conference on Data networks, communications, computers, (2009) November 07-09, pp. 54-59, Baltimore, MD, USA.

[8] U. J. Bora and M. Ahmed, "E-Learning using Cloud Computing", International Journal of Science and Modern Engineering, vol. 1, no. 2, (2013), pp. 9-12.

[9] M. Al-Zoube, S. A. El-Seoud and M. F. Wyne, "Cloud Computing Based E-Learning System", International Journal of Distance Education Technologies, vol. 8, no. $2,(\mathbf{2 0 1 0})$, pp. 58-71.

[10] Whitepaper-Cabling Installation and maintenance. Of wireless, June 2006 edition of Cabling Installation, PennWell Corporation.

[11] Sourangsu Banerji, Rahul Singha Chowdhury, “ On IEEE 802.11: Wireless LAN Technology", International Journal of Mobile Network Communications \& Telematics (IJMNCT) Vol. 3, Issue. 4, 2013. [DOI: 10.5121/ijmnct.2013.3405]

[12] Dilip Antony Joseph, B. S. Manoj, C. Siva Ram Murthyy, "Interoperability of WiFi Hotspots and Cellular Networks", WMASH'04, October 1, 2004, Philadelphia, Pennsylvania, USA.Pp.127-136.

[13] V. Sekar, B. S. Manoj, and C. Siva Ram Murthy, Routing for a Single Interface MCN Architectureand Pricing Schemes for Data Traffic in Multihop Cellular Networks" in Proc. IEEE ICC 2003, Pp. 969-973, May 2003.

[14] Y. D. Lin and Y. C. Hsu, IMultihop Cellular: A New Architecture for Wireless Communications," in Proc. IEEE INFOCOM 2000, pp. 1273-1282, March 2000.

[15] Udeze C.C, Okafor K.C, H. C Inyiama, C.C.Okezie, "A Conceptual Design Model for High Performance Hotspot Network Infrastructure (GRID WLAN) International 
Journal of Advanced Computer Science and Applications, (IJACSA) Vol. 3, No. 1, 2012”, Pp. 93-99.

[16] N. Sulaiman and C.Y.Yaakub," Investigation on QoS of Campus-Wide Wifi Networks", Journal of Telecommunications, Volume 2, Issue 1, February 2010, Pp.12-16.

[17] Ugwoke F.N, K.C Okafor, I.M.Onwusuru \& Udeze C.C, H.C Inyiama, "Characterization of Hotspot Coverage Plan in 2.4/5Ghz Frequency Band (Nnamdi Azikiwe University, Nigeria, As a Case Study)", Computing, Information Systems, Development Informatics \& Allied Research Journal, Vol. 5 No. 1. March, 2014.Pp.77-94.

[18] A.M.Saliu, M.I. Kolo, M.K. Muhammad, L.A. Nafiu, "Internet Authentication and Billing (Hotspot) System Using Mikrotik Router Operating System", International Journal of Wireless Communications and Mobile Computing 2013; 1(1): 51-57.

[19] W.Asif, M.B. Qasim, S.M. R.Tirmzi, U.M.Khan, "Performance Evaluation of WiMAX (802.16) Using Different Encoding Schemes", (IJCSIS) International Journal of Computer Science and Information Security, Vol. 6, No. 2, 2009.pp.332-336.

[20] M.Azizul Hasan, "Performance Evaluation of WiMAX/IEEE 802.16 OFDM Physical Layer, M.SC thesis submitted to Helsinki University of Technology, Department of Electrical and Communications Engineering Communications Laboratory in June 2007

[21] A. Ebian, M. Shokair, and K. H. Awadalla, "Performance Evaluation of Wimax System Using Convolutional Product Code (CPC)", Progress In Electromagnetics Research C, Vol. 5, 125-133, 2008

[22] VineetaJ. K. Thathagar, V. K. Patel, A. R. Charania, "Performance Evaluation of WiMAX System OFDM Physical Layer for Flat-Fading and Multipath-Fading Channel Environments In International Journal of Engineering Research and Applications (IJERA) Vol. 2, Issue 3, May-Jun 2012, Pp.1426-1432

[23] Anuradha.R.kondelwar, K.D.Kulat, "BER Analysis of Proposed Wimax System in different Channel Environments", International Journal of Emerging Technology and Advanced Engineering Website: Volume 2, Issue 9, Sept. 2012, Pp. 28- 38.

[24] Mohammad Shahajahan and A. Q. M. Abdulla Hes-Shafi Analysis of Propagation Models for WiMAX at $3.5 \mathrm{GHz}$, M.SC thesis at Blekinge Institute of Technology September 2009

[25] M.Sreerama Murty,D.Veeraiah, A.Srinivas Rao, "Performance Evaluation of Wi-Fi comparison with WiMAX Networks", In International Journal of Distributed and Parallel Systems (IJDPS) Vol.3, No.1, January 2012 DOI : 10.5121/ijdps.2012.3127 Pp.321329.
[26] Garima Malik, Ajit Singh, "Performance Evaluation of WiFi and WiMax Using OPNET", International Journal of Advanced Research in Computer Science and Software Engineering", Volume 3, Issue 6, June 2013.Pp.572-579

[27] Whitepaper on WiMAX and WiFi Together: Deployment Models and User Scenarios Co-authored by Motorola and Intel

[28] Sumant Ku Mohapatra, Ramya Ranjan Choudhury, Pravanjan Das, "The Future Directions in Evolving WiFi: Technologies, Applications and Services", International Journal of Next-Generation Networks (IJNGN) Vol.6, No.3, September 2014 DOI : 10.5121/ijngn.2014.6302 Pp.13-22

[29] Twenty experts define cloud computing. http://cloudcomputing.sys-con.com/ read/612375_p.htm (18.07.08).

[30] G.F. Pfister, In Search of Clusters, 2nd ed., Prentice Hall, Upper Saddle River, USA, 1998.

[31] in: R. Buyya (Ed.), High Performance Cluster Computing: Architectures and Systems, vol. 1, Prentice Hall, Upper Saddle River, USA, 1999.

[32] R.Buyya, C.S.Yeo, S. Venugopal, J.Broberg, I.Brandic, "Cloud computing and emerging IT platforms: Vision, hype, and reality for delivering computing as the 5th utility", Future Generation Computer Systems 25 (2009) 599_616, www.elsevier.com/locate/fgcs.

[33]] K.C. Okafor, G.N.Ezeh, I.E.Achumba,O.U.Oparaku, U.Diala, "DCCN: A Non-Recursively Built Data Center Architecture for Enterprise Energy Tracking Analytic Cloud Portal, (EEATCP), In AASCIT Computational and Applied Mathematics Journal, 2015, USA. Pp.107-121. Indexed.

[34] T.Ghafarian, H.Deldari, B.Javadi, M.H. Yaghmaeea, R.Buyya, "CycloidGrid: A proximity-aware P2P-based resource discovery architecture in volunteer computing systems", Future Generation Computer Systems 29 (2013) 1583-1595. Available at SciVerse ScienceDirect.

[35] C. Vecchiola, X. Chu, R. Buyya, "Aneka: a software platform for .NET-based Cloud computing," in High Performance \& Large Scale Computing, Advances in Parallel Computing, W. Gentzsch, L. Grandinetti, G. Joubert Eds., IOS Press, 2009.

[36] C. E. Catlett, "TeraGrid: A Foundation for US Cyberinfrastructure," in Network and Parallel Computing, LCNS.Vol. 3779, H. Jin, D. Reed, and W. Jiang Eds., Springer Berlin / Heidelberg, 2005.

[37] F. Gagliardi, M.E. Begin, "EGEE - Providing a Production Quality Grid for e-science," Local to Global Data Interoperability - Challenges and Technologies, Sardinia, Italy, June, 2005 